\title{
ON REDUCIBILITY OF ULTRAMETRIC ALMOST PERIODIC LINEAR REPRESENTATIONS
}

\author{
by BERTIN DIARRA
}

(Received 28 June, 1993)

\begin{abstract}
Let $G$ be a group and $K$ be a complete ultrametric valued field. Let $A P(G, K)$ be the algebra of the generalized almost periodic functions of $G$ in $K$. We have shown in a previous paper that when $A P(G, K)$ has an invariant mean, then any almost periodic linear representation is quasi-reducible. Here, we show that with the same hypothesis, any topologically irreducible almost periodic linear representation is finite dimensional; also, any almost periodic linear representation is the topological sum of irreducible representations. Furthermore, we obtain a Peter-Weyl theorem for the algebra $A P(G, K)$.

We use the technical tools of Hopf algebra theory.
\end{abstract}

\section{Notations and definitions.}

1.1. Almost periodic functions; almost periodic linear representations. Let $G$ be a group and $K$ be a complete ultrametric valued field. The Banach algebra of bounded functions $f: G \rightarrow K$ with the supremum norm $\|f\|=\sup _{s \in G}|f(s)|$ is denoted by $\mathscr{B}(G, K)$. If $f \in \mathscr{B}(G, K)$ we write $\gamma_{s} f(t)=f\left(s^{-1} t\right), \delta_{s} f(t)=f(t s)$ and $\eta(f)(s)=f\left(s^{-1}\right)$ the left (resp. right) translation operator and the inversion operator.

Let us recall the extension of the notion of almost periodic functions given by Schikhof [8], [9]. A function $f \in \mathscr{B}(G, K)$ is called almost periodic if the set $\Gamma_{f}=\left\{\gamma_{s} f\right.$, $s \in G\}$ is a compactoid of $\mathscr{B}(G, K)$ : that is for $\varepsilon>0$, there exist $f_{1}, \ldots, f_{n}$ in $\mathscr{B}(G, K)$ and if $s \in G$, there exist $\lambda_{1}, \ldots, \lambda_{n} \in K,\left|\lambda_{j}\right| \leq 1$, such that $\left\|\gamma_{s} f-\sum_{j=1}^{n} \lambda_{j} f_{j}\right\|<\varepsilon$. The space $A P(G, K)$ of almost periodic functions is a closed subalgebra of $\mathscr{B}(G, K)$ and is invariant with respect to the left (right) translation and the inversion.

If $E$ and $F$ are ultrametric Banach spaces over $K$, we denote by $E \hat{\otimes} F$ the complete tensor product; that is the completion of $E \otimes F$ with respect to the norm $\|z\|=$ $\operatorname{Inf}_{z=\sum x_{j} \otimes y_{j}}\left(\max _{j}\left\|x_{j}\right\|\left\|y_{j}\right\|\right)$. In the sequel, all Banach spaces are ultrametric.

One defines as above, the space $A P(G, E)$ of almost periodic functions of $G$ with values in the Banach space $E$. Furthermore, $A P(G, K) \otimes E$ is isometrically isomorphic to $A P(G, E)$ via the linear map $\Pi_{E}$ defined by $\Pi_{E}(f \otimes x)(s)=f(s) \cdot x$ (cf. [3]).

We say that a linear representation $U: G \rightarrow \mathscr{L}(E)$ is almost periodic if

(i) $\sup _{s \in G}\left\|U_{s}\right\|<+\infty$,

(ii) for any $x \in E$, the function $T_{x}: G \rightarrow E$ defined by $T_{x}(s)=U_{s}(x)$ is almost periodic.

1.2. Complete Hopf algebras: Banach comodules. Let $(H, m, c, \eta, \sigma)$ be a complete ultrametric Hopf algebra over $K, e$ the unit of $H$ and $k$ the canonical map of $K$ in $H$. In other words, $H$ is a Banach algebra with multiplication $m: H \hat{\otimes} H \rightarrow H$; coproduct 
$c: H \otimes H \rightarrow H$ a continuous algebra homomorphism; inversion or antipode $\eta: H \rightarrow H$ a continuous linear map and the counit $\sigma: H \rightarrow K$ a continuous algebra homomorphism. The coassociativity and counitary axioms hold, and $m \circ\left(1_{H} \otimes \eta\right) \circ c=k \circ \sigma=m \circ\left(\eta \otimes 1_{H}\right) \circ \mathrm{c}$.

One sees that $\eta$ is an anti-endomorphism of the algebra (resp. coalgebra) $H$.

ExAmple. The algebra $A P(G, K)$ is a complete Hopf algebra with coproduct $c$ such that $\Pi \circ c(f)(s, t)=f(s t)$; inversion $\eta$ defined by $\eta(f)(s)=f\left(s^{-1}\right)$ and counit $\sigma$ defined by $\sigma(f)=f(e)$, where $e$ is the neutral element of $G$. In fact, $A P(G, K)$ is a complete dual Hopf algebra (cf. [2]).

Let $H^{\prime}$ be the Banach space dual of $H$; if we set for $\mu, v \in H^{\prime}, \mu * v=(\mu \otimes v){ }^{\circ} \mathrm{C}$, then $H^{\prime}$ becomes a complete normed algebra with unit $\sigma$.

A Banach space $E$ is said a left Banach $H$-comodule if there exists a continuous linear map $\Delta: E \rightarrow H \hat{\otimes} E$, called a coproduct, such that $\left(c \otimes 1_{E}\right) \circ \Delta=\left(1_{H} \otimes \Delta\right) \circ \Delta$ and $\left(\sigma \otimes 1_{E}\right) \circ$ $\Delta=1_{E}$. A closed linear subspace $M$ of $E$ is a Banach subcomodule if $\Delta(M) \subset H \hat{\otimes} M$.

Notice that $\|x\| \leq\|\Delta(x)\| \leq\|\Delta\|\|x\|, x \in E$.

Example. Let $E$ be a left Banach $A P(G, K)$-comodule of coproduct $\Delta$. If we set $\varepsilon_{s}(f)=f(s)$ the evaluation map at $s \in G$, then $U_{s}^{\Delta}=\left(\varepsilon_{s^{-1}} \otimes 1_{E}\right) \circ \Delta$ defines an almost periodic (a.p.) linear representation of $G$ in $E$. Conversely, let $U: G \rightarrow \mathscr{L}(E)$ be an a.p. linear representation. If $d_{U}$ denotes the linear map of $E$ in $A P(G, E)$ defined by $d_{U}(x)(s)=U_{s^{-1}}(x)$, then $\Delta_{U}=\Pi_{E}^{-1} \circ d_{U}$ is a coproduct of $E$ and $E$ is a left Banach $A P(G, K)$-comodule. These correspondences are reciprocical (cf. [3]).

If $E$ is a left Banach $H$-comodule, and we set $\mu \cdot x=\left(\mu \otimes 1_{E}\right) \circ \Delta(x)$, for $\mu \in H^{\prime}$, $x \in E$, one induces on $E$ a complete normed right $H^{\prime}$-module structure.

Let us recall that a Banach space $V$ over $K$ is pseudo-reflexive if the canonical map of $V$ into its bidual space $V^{\prime \prime}$ is isometric. It is well known that any linear subspace of a pseudo-reflexive space is pseudo-reflexive.

Also, any Banach space which is a dual space is pseudo-reflexive. It follows that $\mathscr{B}(G, K), A P(G, K)$ and its linear subspaces are pseudo-reflexive. Furthermore let $D$ be a finite dimensional subspace of the pseudo-reflexive space $V$ and $0<\alpha<1$; then for every $d^{\prime} \in D^{\prime}$ there exists $v^{\prime} \in V^{\prime}$ such that $v_{\mid D}^{\prime}=d^{\prime}$ and $\left\|v^{\prime}\right\| \leq \frac{1}{\alpha}\left\|d^{\prime}\right\|$ (cf. [5] or [7]).

THEOREM 1. Let $H$ be a complete ultrametric Hopf algebra that is a pseudo-reflexive Banach space and let $E$ be a left Banach $H$-comodule of coproduct $\Delta$.

(i) A closed linear subspace $M$ of $E$ is a left Banach $H$-subcomodule of $E$ if and only if $M$ is a complete right $H^{\prime}$-submodule of $E$.

(ii) Let $x \in E$; the closure $M_{x}$ of $H^{\prime} \cdot x$ in $E$ is a Banach $H$-subcomodule of $E$ that contains $x$ and is a Banach space of countable type.

Proof. (i) If $M$ is a left Banach subcomodule of $E$, then for $x \in M, \Delta(x) \in H \otimes M$, and if $\mu \in H^{\prime}$, then $\mu \cdot x=\left(\mu \otimes 1_{E}\right) \circ \Delta(x) \in K \otimes M=M$.

On the other hand, if $M$ is a complete right $H^{\prime}$-submodule of $E$, then $\mu \cdot x=$ 
$\left(\mu \otimes 1_{E}\right) \circ \Delta(x) \in M$ for all $\mu \in H^{\prime}$ and $x \in M$. Since $\Delta(x) \in H \hat{\otimes} E$, we can write $\Delta(x)=\sum_{j \geq 1} a_{j} \otimes x_{j}$ where $\left(a_{j}\right)_{j \geq 1}$ is an $\alpha$-orthogonal set of $H, \quad\left(x_{j}\right)_{j \geq 1} \subset E$ and $\alpha \sup _{j \geq 1}\left\|a_{j}\right\|\left\|x_{j}\right\| \leq\|\Delta(x)\| \leq \sup _{j \geq 1}\left\|a_{j}\right\|\left\|x_{j}\right\|$. Hence $\mu \cdot x=\sum_{j \geq 1}\left\langle\mu, a_{j}\right\rangle x_{j} \in M$ for all $\mu \in H^{\prime}$.

Let $\ell$ be an integer $\geq 1$; for $n \geq l+1$ the subspace of $H$ of dimension $n, H_{n}=\bigoplus_{j=1}^{n} K a_{j}$ contains $a_{\rho}$. Let $a_{n \ell}^{\prime}$ be the linear form on $H_{n}$ defined by $\left\langle a_{n \ell}^{\prime}, a_{j}\right\rangle=\delta_{\ell j}, 1 \leq j \leq n$; then $\frac{1}{\left\|a_{e}\right\|} \leq\left\|a_{n \ell}^{\prime}\right\| \leq \frac{1}{\alpha\left\|a_{e}\right\|}$. Since $H$ is a pseudo-reflexive Banach space, there exists $\mu_{n \ell} \in H^{\prime}$

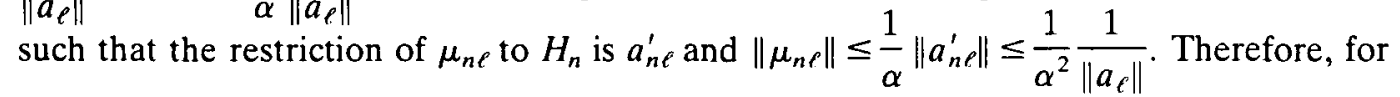
every $n \geq \ell+1, \mu_{n \ell} \cdot x=\sum_{j \geq 1}\left\langle\mu_{n \ell}, a_{j}\right\rangle x_{j}=x_{\ell}+\sum_{j \geq n+1}\left\langle\mu_{n \ell}, a_{j}\right\rangle x_{j} \in M$. However

$$
\left\|\sum_{j \geq n+1}\left\langle\mu_{n \ell}, a_{j}\right\rangle x_{j}\right\| \leq \sup _{j \geq n+1}\left\|\mu_{n \ell}\right\|\left\|a_{j}\right\|\left\|x_{j}\right\| \leq \frac{1}{\alpha^{2}} \frac{1}{\left\|a_{\ell}\right\|} \sup _{j \geq n+1}\left\|a_{j}\right\|\left\|x_{j}\right\|
$$

and $\lim _{n \rightarrow+\infty} \sup _{j \geq n+1}\left\|a_{j}\right\|\left\|x_{j}\right\|=0$. It follows that $x_{\ell}=\lim _{n \rightarrow+\infty} \mu_{n \ell} \cdot x \in M$ and $\Delta(x)=\sum_{l \geq 1} a_{\ell} \otimes x_{\ell}$ $\in H \otimes M$. That is $M$ is a Banach subcomodule of $E$.

(ii) Let $x \in E$; it is clear that $H^{\prime} \cdot x$ contains $x$, furthermore $\mu \cdot(v \cdot x)=(v * \mu) \cdot x \in$ $H^{\prime} \cdot x$ for all $\mu, v \in H^{\prime}$; hence the closure $\overline{H^{\prime} \cdot x}=M_{x}$ is a complete right $H^{\prime}$-submodule of $E$.

With the same notations as in (i) we have $\Delta(x)=\sum_{j \geq 1} a_{j} \otimes x_{j}$. Let $E_{0}=$ $E\left[x_{1}, \ldots, x_{j}, \ldots\right]$ be the closed linear subspace of $E$ spanned by $\left(x_{j}\right)_{j \geq 1}$. First, it is clear that $E_{0}$ is a Banach space of countable type. On the other hand, if $\mu \in H^{\prime}$, we have $\mu \cdot x=\sum_{j \geq 1}\left\langle\mu, a_{j}\right\rangle x_{j} \in E_{0}$; hence $H^{\prime} \cdot x \subset E_{0}$ and $M_{x}=\overline{H^{\prime} \cdot x} \subset E_{0}$.

Since $M_{x}$ is a closed right $H^{\prime}$-submodule of $E$, we deduce from (i) that $M_{x}$ is a left Banach $H$-subcomodule of $E$ and that $x_{j} \in M_{x}, j \geq 1$. Hence $M_{x}=E_{0}$ is a Banach space of countable type.

Note. The theorem, applied to an a.p. linear representation $U$ of $G$ in $E$, shows that if $x \in E$, the closed linear subspace of $E$ spanned by $C_{x}=\left\{U_{s}(x), s \in G\right\}$ is of countable type. As observed in [3], this also follows from the fact that $C_{x}$ is a compactoid of $E$.

\section{Banach comodule morphisms.}

II.1. Definition. Let $E$ and $F$ be two left Banach $H$-comodules of coproducts $\Delta_{E}$ and $\Delta_{F}$ respectively. A continuous linear map $u: E \rightarrow F$ is a Banach comodule morphism if $\Delta_{F} \circ u=\left(1_{H} \otimes u\right) \circ \Delta_{E}$.

Lemma 1. Let $u: E \rightarrow F$ be a Banach comodule morphism.

(i) If $V$ is a Banach subcomodule of $F$, then $u^{-1}(V)$ is a Banach subcomodule of $E$.

(ii) The closure $\overline{u(E)}$ of $u(E)$ is a Banach subcomodule of $F$. 
Proof. (i) Indeed, for $x \in u^{-1}(V), \Delta_{E}(x)=\sum_{j \geq 1} a_{j} \otimes x_{j}$ where $\left(a_{j}\right)_{j \geq 1}$ is an $\alpha$-orthogonal set of $H$ and $\left(x_{j}\right)_{j \geq 1} \subset E$. Since $\Delta_{F}(V) \subset H \hat{\otimes} V$ and $u(x) \in V$, we have $\Delta_{F}(u(x))=$ $\sum_{\ell \geq 1} b_{\ell} \otimes y_{\ell}$, where $\left(b_{\ell}\right)_{\ell \geq 1} \subset H$ and $\left(y_{\ell}\right)_{l \geq 1} \subset V$.

Let $H_{0}=E\left[a_{1}, \ldots, a_{j}, \ldots ; b_{1}, \ldots, b_{\ell}, \ldots\right]$ be the closed subspace of $H$ spanned by $\left\{a_{j}, j \geq 1 ; b_{\ell}, \ell \geq 1\right\}$. This Banach space is of countable type. If $H_{1}=E\left[a_{1}, \ldots, a_{j}, \ldots\right]$ is the closed subspace of $H$ spanned by $\left(a_{j}\right)_{j \geq 1}$, then there exists a continuous linear projection $p$ of $H_{0}$ onto $H_{1}$ such that $\|p\| \leq \frac{1}{\alpha}$ (cf. [7]). Let $a_{j}^{\prime} \in H_{1}^{\prime}$ be defined by $\left\langle a_{j}^{\prime}, a_{t}\right\rangle=\delta_{j t}, t \geq 1$ and put $\tilde{a}_{j}^{\prime}=a_{j}^{\prime} \circ p \in H_{0}^{\prime}$. Then $\left(\tilde{a}_{j}^{\prime} \otimes 1_{F}\right) \circ \Delta_{F}(u(x))=\sum_{\ell \geq 1}\left\langle\tilde{a}_{j}^{\prime}, b_{\ell}\right\rangle y_{\ell}=\left(\tilde{a}_{j}^{\prime} \otimes 1_{F}\right) \circ\left(1_{H} \otimes u\right) \circ \Delta_{E}(x)$ $=\sum_{t \geq 1}\left\langle\tilde{a}_{j}^{\prime}, a_{t}\right\rangle u\left(x_{t}\right)=\sum_{t \geq 1} \delta_{j t} u\left(x_{t}\right)=u\left(x_{j}\right)$. Therefore $u\left(x_{j}\right)=\sum_{l \geq 1}\left\langle\tilde{a}_{j}^{\prime}, b_{\ell}\right\rangle y_{\ell} \in V ;$ hence $x_{j} \in$ $u^{-1}(V)$ and $\Delta_{E}(x)=\sum_{j \geq 1} a_{j} \otimes x_{j} \in H \otimes u^{-1}(V)$.

(ii) For $z=u(x)$ in $u(E), \quad \Delta_{F}(z)=\Delta_{F}(u(x))=\left(1_{H} \otimes u\right) \circ \Delta_{E}(x)=\sum_{j \geq 1} a_{j} \otimes u\left(x_{j}\right) \epsilon$ $H \hat{\otimes} \overline{u(E)}$. Therefore $\Delta_{F}(u(E)) \subset H \hat{\otimes} \overline{u(E)}$. However $\Delta_{F}$ is a homeomorphism of $F$ onto $\Delta_{F}(F)$; hence $\Delta_{F}\left(\overline{u(E))}=\overline{\Delta_{F}(u(E))} \subset H \hat{\otimes} \overline{u(E)}\right.$.

Corollary. Let $V$ and $W$ be Banach subcomodules of the left Banach H-comodule $E$; then $V \cap W$ is a Banach subcomodule of $E$.

Proof. (a)- Although a direct proof of this corollary is easy, we have the opportunity to define the direct sum of a finite family $\left(E_{i}, \Delta_{i}\right)_{1 \leq i \leq n}$ of left Banach $H$-comodules as follows. Let $E=\bigoplus_{i=1}^{n} E_{i}$, equipped with a norm equivalent to the norm $\left\|\sum_{i=1}^{n} x_{i}\right\|=\max _{1 \leq i \leq n}\left\|x_{i}\right\|$. Put $\Delta=\bigoplus_{i=1}^{n} \Delta_{i}$; i.e. $\Delta\left(\sum_{i=1}^{n} x_{i}\right)=\sum_{i=1}^{n} \Delta_{i}\left(x_{i}\right)$. It is readily seen that $(E, \Delta)$ is a left Banach $H$-comodule.

(b)- Put $F=E \oplus E$ and $\Delta_{F}=\Delta_{E} \oplus \Delta_{E}$; then $V \oplus W$ is a Banach subcomodule of $F$ if $V$ and $W$ are Banach subcomodules of $E$. The continuous linear injective map $u$ of $E$ into $F$ defined by $u(x)=x \oplus x$ is a Banach comodule morphism. Thus $V \cap W=u^{-1}(V \oplus W)$ is a Banach subcomodule of $E$.

II.2. Spaces of Banach comodule morphisms. Let us recall that a continuous linear operator $u: E \rightarrow F$ is completely continuous if $u=\lim _{n \rightarrow+\infty} u_{n}$, where $u_{n}: E \rightarrow F$ is linear continuous of finite rank. Furthermore, the space $C(E, F)$ of completely continuous operators is closed in $\mathscr{L}(E, F)$ and is isometrically isomorphic to $E^{\prime} \hat{\otimes} F$.

If $E$ and $F$ are left Banach $H$-comodules we denote by $\operatorname{Hom}_{\text {com }}(E, F)$ the set of Banach comodule morphisms. We set $C_{\text {com }}(E, F)=C(E, F) \cap \operatorname{Hom}_{\text {com }}(E, F)$ and $\operatorname{End}_{\text {com }}(E)=\operatorname{Hom}_{\text {com }}(E, E) ; C_{\text {com }}(E)=C_{\text {com }}(E, E)$.

Proposition 1. Let $E, F$ and $L$ be three left Banach $H$-comodules. 
(i) If $u: E \rightarrow F$ and $v: F \rightarrow L$ are Banach comodule morphisms, then $v \circ u: E \rightarrow L$ is a Banach comodule morphism.

(ii) $\mathrm{Hom}_{\mathrm{com}}(E, F)$ [resp. End $\mathrm{com}(E)$ ] is a Banach space [resp. a unitary Banach algebra]. Furthermore $C_{\mathrm{com}}(E, F)\left[\right.$ resp. $\left.C_{\mathrm{com}}(E)\right]$ is a closed linear subspace [resp. a closed two-sided ideal] of $\operatorname{Hom}_{\mathrm{com}}(E, F)$ [resp. $\left.\operatorname{End}_{\mathrm{com}}(E)\right]$.

Proof. It is easy. For instance $\Delta_{L} \circ(v \circ u)=\left(\Delta_{L} \circ v\right) \circ u=\left(1_{H} \otimes v\right) \circ \Delta_{F} \circ u=\left(1_{H} \otimes v\right) \circ$ $\left(1_{H} \otimes u\right) \circ \Delta_{E}=\left[1_{H} \otimes(v \circ u)\right] \circ \Delta_{E}$. Also, if $u=\lim _{n \rightarrow+\infty} u_{n}$ with $u_{n} \in \operatorname{Hom}_{\mathrm{com}}(E, F)$, then $\Delta_{F} \circ u=\Delta_{F} \circ\left(\lim _{n \rightarrow+\infty} u_{n}\right)=\lim _{n \rightarrow+\infty} \Delta_{F} \circ u_{n}=\lim _{n \rightarrow+\infty}\left(1_{H} \otimes u_{n}\right) \circ \Delta_{E}=\left(1_{H} \otimes u\right) \circ \Delta_{E}$.

Corollary. Let $u \in \operatorname{End}_{\text {com }}(E)$ [resp. $\left.C_{\text {com }}(E)\right]$ if $S=\sum_{n \geq 0} \lambda_{n} X^{n}$ is a formal power series with coefficients in $K$ [resp. and $\left.\lambda_{0}=0\right]$ such that $S(u)=\sum_{n \geq 0} \lambda_{n} u^{n}$ is converging in $\mathscr{L}(E)$, then $S(u) \in \operatorname{End}_{\text {com }}(E)\left[\right.$ resp. $\left.C_{\text {com }}(E)\right]$.

\section{3. When $\boldsymbol{H}$ admits a left integral.}

II.3.1. Banach comodule morphism associated with a linear map. By definition, a left integral for the complete Hopf algebra is an element $v$ of $H^{\prime}$ such that $\mu * v=\langle\mu, e\rangle \nu$, for all $\mu \in H^{\prime}$.

Assume that the duality $\left\langle H^{\prime}, H\right\rangle$ is separated; then $v \in H^{\prime}$ is a left integral for $H$ if and only if $\left(1_{H} \otimes v\right) \circ c=k \circ v$.

In the sequel, we suppose that $H$ admits a left integral $v$ such that $\langle v, e\rangle=1$. Hence the continuous linear form $\varphi=v \circ m^{\circ}\left(1_{H} \otimes \eta\right): H \hat{\otimes} H \rightarrow K$ satisfies: (i) $\varphi^{\circ} c=\sigma$ and (ii) $\left(\varphi \otimes 1_{H}\right) \circ\left(1_{H} \otimes c\right)=\left(1_{H} \otimes \varphi\right) \circ\left(c \otimes 1_{H}\right)$. Furthermore $\varphi(a \otimes e)=v(a)$ and $\|v\| \leq\|\varphi\| \leq$ $\|v\|\|\eta\|$.

Let $E$ and $F$ be two left Banach $H$-comodules of coproducts $\Delta_{E}$ and $\Delta_{F}$. If $u: E \rightarrow F$ is a continuous linear map, we put as in group representations theory

$$
u^{\#}=\left(\varphi \otimes 1_{F}\right) \circ\left(1_{H} \otimes \Delta_{F}\right) \circ\left(1_{H} \otimes u\right) \circ \Delta_{E} ;
$$

hence $u^{\#}: E \rightarrow F$ is linear and continuous.

Proposition 2. Let $E$ and $F$ be two left Banach comodules and $u: E \rightarrow F$ be a continuous linear map.

(i) $u^{\#}$ is a Banach comodule morphism.

(ii) The map $u \rightarrow u^{\#}$ of $\mathscr{L}(E, F)$ into $\operatorname{Hom}_{\text {com }}(E, F)$ is linear and continuous. Moreover, $u^{\# \#}=u^{\#}$ and $u$ is a Banach comodule morphism if and only if $u^{\#}=u$.

Proof: (i) One verifies that

$1_{H} \otimes\left[\left(\varphi \otimes 1_{F}\right) \circ\left(1_{H} \otimes \Delta_{F}\right) \circ\left(1_{H} \otimes u\right) \circ \Delta_{E}\right]=$

$$
\left(1_{H} \otimes \varphi \otimes 1_{F}\right) \circ\left(1_{H} \otimes 1_{H} \otimes \Delta_{F}\right) \circ\left(1_{H} \otimes 1_{H} \otimes u\right) \circ\left(1_{H} \otimes \Delta_{E}\right)
$$


Hence, one sees that

$$
\begin{aligned}
& \left(1_{H} \otimes u^{\#}\right) \circ \Delta_{E}=\left(1_{H} \otimes\left[\left(\varphi \otimes 1_{F}\right) \circ\left(1_{H} \otimes \Delta_{F}\right) \circ\left(1_{H} \otimes u\right) \circ \Delta_{E}\right]\right) \circ \Delta_{E}= \\
& =\left(1_{H} \otimes \varphi \otimes 1_{F}\right) \circ\left(1_{H} \otimes 1_{H} \otimes \Delta_{F}\right) \circ\left(1_{H} \otimes 1_{H} \otimes u\right) \circ\left(1_{H} \otimes \Delta_{E}\right) \circ \Delta_{E}= \\
& =\left(1_{H} \otimes \varphi \otimes 1_{F}\right) \circ\left(1_{H} \otimes 1_{H} \otimes \Delta_{F}\right) \circ\left(1_{H} \otimes 1_{H} \otimes u\right) \circ\left(c \otimes 1_{E}\right) \circ \Delta_{E}= \\
& =\left(1_{H} \otimes \varphi \otimes 1_{F}\right) \circ\left(c \otimes 1_{H} \otimes 1_{F}\right) \circ\left(1_{H} \otimes \Delta_{F}\right) \circ\left(1_{H} \otimes u\right) \circ \Delta_{E}= \\
& =\left(\varphi \otimes 1_{H} \otimes 1_{F}\right) \circ\left[1_{H} \otimes\left(c \otimes 1_{F}\right) \circ \Delta_{F}\right] \circ\left(1_{H} \otimes u\right) \circ \Delta_{E}= \\
& =\left(\varphi \otimes 1_{H} \otimes 1_{F}\right) \circ\left(1_{H} \otimes 1_{H} \otimes \Delta_{F}\right) \circ\left(1_{H} \otimes \Delta_{F}\right) \circ\left(1_{H} \otimes u\right) \circ \Delta_{E} .
\end{aligned}
$$

However $\left(\varphi \otimes 1_{H} \otimes 1_{F}\right) \circ\left(1_{H} \otimes 1_{H} \otimes \Delta_{F}\right)=\Delta_{F} \circ\left(\varphi \otimes 1_{F}\right)$. Therefore, $\left(1_{H} \otimes u^{\#}\right) \circ \Delta_{E}=$ $\Delta_{F} \circ\left(\varphi \otimes 1_{F}\right) \circ\left(1_{H} \otimes \Delta_{F}\right) \circ\left(1_{H} \otimes u\right) \circ \Delta_{E}=\Delta_{F} \circ u^{\#}$; i.e. $u^{\#}$ is a comodule morphism.

(ii) It is readily seen that $u \rightarrow u^{\#}$ is linear and continuous with norm $\leq\|\varphi\|\left\|\Delta_{E}\right\|\left\|\Delta_{F}\right\|$.

If $u$ is a comodule morphism, one sees that

$$
\begin{aligned}
u^{\#} & =\left(\varphi \otimes 1_{F}\right) \circ\left(1_{H} \otimes \Delta_{F}\right) \circ\left(1_{H} \otimes u\right) \circ \Delta_{E}=\left(\varphi \otimes 1_{F}\right) \circ\left(1_{H} \otimes \Delta_{F}\right) \circ \Delta_{F} \circ u \\
& =\left(\varphi \otimes 1_{F}\right) \circ\left(c \otimes 1_{F}\right) \circ \Delta_{F} \circ u=\left(\varphi \circ c \otimes 1_{F}\right) \circ \Delta_{F} \circ u=\left[\left(\sigma \otimes 1_{F}\right) \circ \Delta_{F}\right] \circ u=u .
\end{aligned}
$$

Conversely, if $u=u^{\#}$, from (i) it follows that $u$ is a comodule morphism. Hence, for any continuous linear map $u: E \rightarrow F$, one has $u^{\# \#}=u^{\#}$.

Lemma 2. Let $E, F$ and $L$ be three left Banach $H$-comodules, $u: E \rightarrow F$ and $v: F \rightarrow L$ be continuous linear maps. Then $\left(v \circ u^{\#}\right)^{\#}=v^{\#} \circ u^{\#}$

Proof. Obviously, $\quad\left(v \circ u^{\#}\right)^{\#}=\left(\varphi \otimes 1_{L}\right) \circ\left(1_{H} \otimes \Delta_{L}\right) \circ\left(1_{H} \otimes v \circ u^{\#}\right) \circ \Delta_{E}=\left(\varphi \otimes 1_{L}\right) \circ$ $\left(1_{H} \otimes \Delta_{L}\right) \circ\left(1_{H} \otimes v\right) \circ\left(1_{H} \otimes u^{\#}\right) \circ \Delta_{E}=\left(\varphi \otimes 1_{L}\right) \circ\left(1_{H} \otimes \Delta_{L}\right) \circ\left(1_{H} \otimes v\right) \circ \Delta_{F} \circ u^{\#}=v^{\#} \circ u^{\#}$.

COROLlary. Let $E$ be a left Banach $H$-comodule. If $p: E \rightarrow E$ is a continuous linear projection of $E$ onto $M=p(E)$ and if $M$ is a Banach subcomodule of $E$; then $p^{\#}$ is a projection of $E$ onto $M$ and $E=M \oplus N$, a direct sum of Banach comodules, where $N=\operatorname{ker} p^{\#}$.

Proof. Put $\Delta_{E}=\Delta$. By hypothesis, for any $y \in M, \Delta(y)=\sum_{\ell \geq 1} b_{\ell} \otimes y_{\ell} \in H \hat{\otimes} M$. Let $x \in E$; setting $\Delta(x)=\sum_{j \geq 1} a_{j} \otimes x_{j} \in H \hat{\otimes} E$, it follows that

$p^{\#}(x)=\left(\varphi \otimes 1_{E}\right) \circ\left(1_{H} \otimes \Delta\right) \circ\left(1_{H} \otimes p\right) \circ \Delta(x)$

$$
=\left(\varphi \otimes 1_{E}\right)\left(\sum_{j \geq 1} a_{j} \otimes \Delta \circ p\left(x_{j}\right)\right)=\sum_{j \geq 1} \sum_{\ell \geq 1} \varphi\left(a_{j} \otimes b_{\ell j}\right) y_{\ell j}
$$

with $y_{\ell j} \in M$. Since $y_{\ell j}=p\left(x_{\ell j}\right)$, we have

$$
p^{\#}(x)=p\left(\sum_{j \geq 1} \sum_{\ell \geq 1} \varphi\left(a_{j} \otimes b_{\ell_{j}}\right) x_{\ell_{j}}\right)=p(z) \in p(E)=M ;
$$

i.e. $p^{\#}(E) \subset M$. 
On the other hand, since for any $y \in M, p(y)=y$, one has

$$
\begin{aligned}
p^{\#}(y)=\left(\varphi \otimes 1_{E}\right)\left(\sum_{l \geq 1} b_{\ell} \otimes \Delta\left(y_{\ell}\right)\right)=\left(\varphi \otimes 1_{E}\right) \circ\left(1_{H} \otimes \Delta\right) \circ \Delta(y) \\
=\left(\varphi \otimes 1_{E}\right) \circ\left(c \otimes 1_{E}\right) \circ \Delta(y)=\left(\varphi \circ c \otimes 1_{E}\right) \circ \Delta(y)=\left(\sigma \otimes 1_{E}\right) \circ \Delta(y)=y .
\end{aligned}
$$

Therefore $M \subset p^{\#}(M) \subset p^{\#}(E)$. We have proved that $M=p^{\#}(E)$.

Since for any $x \in E, p^{\#}(x)=p(z)$, one has $p^{\circ} p^{\#}(x)=p^{\circ} p(z)=p(z)=p^{\#}(x)$; in

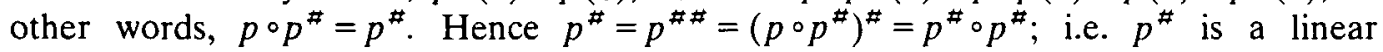
projection as well as a comodule morphism of $E$ onto $M$. The corollary is proved. [3].

NOTE. This corollary gives a proof of the implication (iv) $\Rightarrow$ (i) of the Theorem 3 in

Proposition 3. Let $E$ and $F$ be two left Banach $H$-comodules and let $u: E \rightarrow F$ be $a$ completely continuous operator. Then $u^{\#}$ is completely continuous.

Proof. (i) Since the map $u \rightarrow u^{\#}$ is linear and continuous, if $u=\sum_{n \geq 1} x_{n}^{\prime} \otimes z_{n} \in$ $C(E, F)=E^{\prime} \hat{\otimes} F$, one has, $u^{\#}=\sum_{n \geq 1}\left(x_{n}^{\prime} \otimes z_{n}\right)^{\#}$ in $\operatorname{Hom}_{\text {com }}(E, F)$. But $C(E, F)$ is a Banach space; hence, it suffices to prove that for any $x^{\prime} \in E^{\prime}$ and any $z \in F$, one has $\left(x^{\prime} \otimes z\right)^{\#} \in C(E, F)$.

(ii) Put $u=x^{\prime} \otimes z$. First, $\Delta_{F}(z)=\sum_{\ell \geq 1} b_{\ell} \otimes z_{\ell}$, where $\left(b_{\ell}\right)_{\ell \geq 1} \subset H$ and $\left(z_{\ell}\right)_{\ell \geq 1}$ is an $\alpha$-orthogonal set of $F$, with

$$
\alpha \sup _{\ell \geq 1}\left\|b_{\ell}\right\|\left\|z_{\ell}\right\| \leq\left\|\Delta_{F}(z)\right\| \leq \sup _{\ell \geq 1}\left\|b_{\ell}\right\|\left\|z_{\ell}\right\| .
$$

Also, for $x \in E, \Delta_{E}(x)=\sum_{j \geq 1} a_{j} \otimes x_{j}$, where $\left(a_{j}\right)_{j \geq 1} \subset H,\left(x_{j}\right)_{j \geq 1}$ is an $\alpha$-orthogonal set of $E$ and one has an inequality similar to (0). On the other hand,

$$
\begin{aligned}
& u^{\#}(x)=\left(\varphi \otimes 1_{F}\right) \circ\left(1_{H} \otimes \Delta_{F}\right) \circ\left(1_{H} \otimes u\right) \circ \Delta_{E}(x) \\
& =\left(\varphi \otimes 1_{F}\right) \circ\left(1_{H} \otimes \Delta_{F}\right)\left(\sum_{j \geq 1} a_{j} \otimes u\left(x_{j}\right)\right)=\left(\varphi \otimes 1_{F}\right)\left(\sum_{j \geq 1} a_{j} \otimes\left\langle x^{\prime}, x_{j}\right\rangle \Delta_{F}(z)\right) \\
& =\sum_{j \geq 1} \sum_{\ell \geq 1}\left\langle x^{\prime}, x_{j}\right\rangle \varphi\left(a_{j} \otimes b_{\ell}\right) z_{\ell} .
\end{aligned}
$$

However $\left\|\left\langle x^{\prime}, x_{j}\right\rangle \varphi\left(a_{j} \otimes b_{\ell}\right) z_{\ell}\right\| \leq\left\|x^{\prime}\right\|\|\varphi\|\left\|a_{j}\right\|\left\|x_{j}\right\|\left\|b_{\ell}\right\|\left\|z_{\ell}\right\|$ with $\lim _{j \rightarrow+\infty}\left\|a_{j}\right\|\left\|x_{j}\right\|=0=$ $\lim _{l \rightarrow+\infty}\left\|b_{\ell}\right\|\left\|z_{\ell}\right\|$. Hence, the family $\left(\left\langle x^{\prime}, x_{j}\right\rangle \varphi\left(a_{j} \otimes b_{\ell}\right) z_{\ell}\right)_{j, \ell}$ is summable. Therefore

$$
u^{\#}(x)=\sum_{\ell \geq 1} \sum_{j \geq 1}\left\langle x^{\prime}, x_{j}\right\rangle \varphi\left(a_{j} \otimes b_{\ell}\right) z_{\ell} .
$$

Let $F_{0}=E\left[z_{1}, \ldots, z_{\ell}, \ldots\right]$ be the closed subspace of $F$ spanned by the $\alpha$-orthogonal set $\left(z_{\ell}\right)_{\ell \geq 1}$. If $z_{\ell}^{\prime} \in F_{0}^{\prime}$ is defined by $\left\langle z_{\ell}^{\prime}, z_{k}\right\rangle=\delta_{\ell k}$, then $\frac{1}{\left\|z_{\ell}\right\|} \leq\left\|z_{\ell}^{\prime}\right\| \leq \frac{1}{\alpha\left\|z_{\ell}\right\|}$. 
It is clear that for any $x \in E, u^{\#}(x) \in F_{0}$ and we obtain the adjoint map ${ }^{\prime} u^{\sharp}: F_{0}^{\prime} \rightarrow E^{\prime}$ with ' $u^{\#}\left(z_{\ell}^{\prime}\right) \in E^{\prime}$ and for any $x \in E$, we have

$$
\left\langle{ }^{\prime} u^{\#}\left(z_{\ell}^{\prime}\right), x\right\rangle=\left\langle z_{\ell}^{\prime}, u^{\#}(x)\right\rangle=\sum_{j \geq 1}\left\langle x^{\prime}, x_{j}\right\rangle \varphi\left(a_{j} \otimes b_{\ell}\right) .
$$

Moreover,

$$
\begin{aligned}
\left.\left.\right|^{\prime} u^{\#}\left(z_{\ell}^{\prime}\right), x\right\rangle \mid & \leq \sup _{j \geq 1}\left|\left\langle x^{\prime}, x_{j}\right\rangle\right| \mid \varphi\left(a_{j} \otimes b_{\ell} \mid \leq\left\|x^{\prime}\right\|\|\varphi\|\left\|b_{\ell}\right\| \sup _{j \geq 1}\left\|a_{j}\right\|\left\|x_{j}\right\|\right. \\
& \leq \frac{1}{\alpha}\left\|x^{\prime}\right\|\|\varphi\|\left\|b_{\ell}\right\|\left\|\Delta_{E}(x)\right\| \leq \frac{1}{\alpha}\left\|x^{\prime}\right\|\|\varphi\|\left\|\Delta_{E}\right\|\left\|b_{\ell}\right\|\|x\| .
\end{aligned}
$$

Hence, we have

$$
\left\|u^{\prime}\left(z_{\ell}^{\prime}\right)\right\| \leq \frac{1}{\alpha}\left\|x^{\prime}\right\|\|\varphi\|\left\|\Delta_{E}\right\|\left\|b_{\ell}\right\|
$$

It follows that

$$
\left\|^{t} u^{\#}\left(z_{\ell}^{\prime}\right) \otimes z_{\ell}\right\| \leq \frac{1}{\alpha}\left\|x^{\prime}\right\|\|\varphi\|\left\|\Delta_{E}\right\|\left\|b_{\ell}\right\|\left\|z_{\ell}\right\| .
$$

Since $\lim _{\ell \rightarrow+\infty}\left\|b_{\ell}\right\|\left\|z_{\ell}\right\|=0$, we have $\lim _{\ell \rightarrow+\infty}\left\|^{i} u^{\ddagger}\left(z_{\ell}^{\prime}\right) \otimes z_{\ell}\right\|=0$ and $\sum_{l \geq 1} u^{\#}\left(z_{\ell}^{\prime}\right) \otimes z_{\ell} \in$ $E^{\prime} \otimes F=C(E, F)$. However for any $x \in E$,

$$
\left(\sum_{\ell \geq 1}{ }^{t} u^{\#}\left(z_{\ell}^{\prime}\right) \otimes z_{\ell}\right)(x)=\sum_{\ell \geq 1}\left\langle{ }^{t} u^{\#}\left(z_{\ell}^{\prime}\right), x\right\rangle z_{\ell}=\sum_{\ell \geq 1} \sum_{j \geq 1}\left\langle x^{\prime}, x_{j}\right\rangle \varphi\left(a_{j} \otimes b_{\ell}\right) z_{\ell}=u^{\#}(x),
$$

by (1). It follows that $u^{\#}=\sum_{\ell \geq 1}{ }^{t} u^{\#}\left(z_{\ell}^{\prime}\right) \otimes z_{\ell} \in C(E, F)$.

REMARK 1. Recall that $v$ is a left integral for $A P(G, K)$ such that $\langle v, 1\rangle \neq 0$ iff $v \neq 0$ and $\left\langle v, \gamma_{s} f\right\rangle=\langle v, f\rangle=\left\langle v, \delta_{s} f\right\rangle$ for all $s \in G$. Moreover, if $\langle v, 1\rangle=1, v$ is called an invariant mean. If $F$ is a Banach space, we have an extension $v_{F}=v \otimes 1_{F}$ of $v$ on $A P(G, F)=$ $A P(G, K) \hat{\oplus} F$ with values in $F$ and $v_{F}\left(\gamma_{s} \varphi\right)=v_{F}(\varphi)=v_{F}\left(\delta_{s} \varphi\right), s \in G$.

For the a.p. linear representations $U: G \rightarrow \mathscr{L}(E), V: G \rightarrow \mathscr{L}(F)$ and the continuous linear map $u: E \rightarrow F$, the intertwining operator (as well as comodule morphism) $u^{\#}$ can be written in the classical form $u^{\#}(x)=\int_{G} V_{s} \circ u \circ U_{s^{-1}}(x) d v_{F}(s)$.

I.3.2. Comodules which are free Banach spaces. Let $E$ be a free Banach space; that is to say, $E$ is isomorphic to a space $c_{0}(I, K)=\left\{\left(\lambda_{j}\right)_{j \in I} \subset K / \lim _{j} \lambda_{j}=0\right\}$. In other words, there exists $\left(e_{j}\right)_{j \in I} \subset E$, called a base of $E$, two real numbers $\alpha_{0}$ and $\alpha_{1}>0$, such that any $x \in E$ can be written in the form $x=\sum_{j \in I} \lambda_{j} e_{j}, \lambda_{j} \in K$ and $\alpha_{0} \sup _{j \in I}\left|\lambda_{j}\right| \leq\|x\| \leq \alpha_{1} \sup _{j \in I}\left|\lambda_{j}\right|$. For the continuous linear form $e_{j}^{\prime} \in E^{\prime}$, defined by $\left\langle e_{j}^{\prime}, e_{\ell}\right\rangle=\delta_{j \ell}$, one has $\frac{1}{\alpha_{1}} \leq\left\|e_{j}^{\prime}\right\| \leq \frac{1}{\alpha_{0}}$. 
Let $F$ be another Banach space. The complete tensor product $F \hat{\otimes} E$ is isomorphic to $c_{0}(I, F)=\left\{\left(y_{i}\right)_{j \in I} \subset F / \lim _{j}\left\|y_{j}\right\|=0\right\}$. In fact, each $z \in F \hat{\otimes} E$ is in the form $z=\sum_{j \in I} y_{j} \otimes e_{j}$, where $y_{j} \in F$ and $\lim _{j}\left\|y_{j}\right\|=0$. Furthermore $\alpha_{0} \sup _{j \in I}\left\|y_{j}\right\| \leq\|z\| \leq \alpha_{1} \sup _{j \in I}\left\|y_{j}\right\|$.

Assume that the free Banach space $E$ is a left Banach $H$-comodule with coproduct $\Delta: E \rightarrow H \hat{\otimes} E$. For $x \in E$, one has $\Delta(x)=\sum_{j \in I} A_{j}(x) \otimes e_{j}$. Hence one defines, for each $j \in I$, a continuous linear map $A_{j}: E \rightarrow H$ and $\alpha_{0} \sup _{j \in I}\left\|A_{j}\right\| \leq\|\Delta\| \leq \alpha_{1} \sup _{j \in I}\left\|A_{j}\right\|$.

Put, for $\ell \in I, A_{j}\left(e_{\ell}\right)=a_{\ell j} \in H$; one has

$$
\Delta\left(e_{\ell}\right)=\sum_{j \in I} a_{\ell j} \otimes e_{j}, \quad \lim _{j} a_{\ell j}=0 .
$$

Note. $A_{j}=\left(1_{H} \otimes e_{j}^{\prime}\right) \circ \Delta$ and $\bigcap_{j \in I} \operatorname{ker} A_{j}=(0)$.

More generally, if $x^{\prime} \in E^{\prime}$, we put $A_{x^{\prime}}=\left(1_{H} \otimes x^{\prime}\right) \circ \Delta$. Obviously, $H$ is a left Banach $H$-comodule with respect to its coproduct $c$.

Lemma 3. For any $x^{\prime} \in E^{\prime}$, the linear map $A_{x^{\prime}}=\left(1_{H} \otimes x^{\prime}\right) \circ \Delta: E \rightarrow H$ is a comodule morphism.

Proof. It is easy to see that $c \circ\left(1_{H} \otimes x^{\prime}\right)=c \otimes x^{\prime}=\left(1_{H} \otimes 1_{H} \otimes x^{\prime}\right) \circ\left(c \otimes 1_{E}\right)$, So $c \circ A_{x^{\prime}}=c \circ\left(1_{H} \otimes x^{\prime}\right) \circ \Delta=\left(1_{H} \otimes 1_{H} \otimes x^{\prime}\right) \circ\left(c \otimes 1_{E}\right) \circ \Delta=\left(1_{H} \otimes 1_{H} \otimes x^{\prime}\right) \circ\left(1_{H} \otimes \Delta\right) \circ \Delta$ $=\left[1_{H} \otimes\left(1_{H} \otimes x^{\prime}\right) \circ \Delta\right] \circ \Delta=\left(1_{H} \otimes A_{x^{\prime}}\right) \circ \Delta$.

Lemma 4. For all $\ell, j \in I$, one has

(i) $c\left(a_{\ell j}\right)=\sum_{n \in I} a_{\ell n} \otimes a_{n j}$,

(ii) $\sigma\left(a_{\ell j}\right)=\delta_{\ell j}$,

(iii) $\sum_{n \in I} a_{e n} \eta\left(a_{n j}\right)=\delta_{\ell j} \cdot e=\sum_{n \in I} \eta\left(a_{e n}\right) a_{n j}$.

Proof. (i) Since $A_{j}=\left(1_{H} \otimes e_{j}^{\prime}\right) \circ \Delta$ is a comodule morphism, we have $c\left(a_{t j}\right)=$ $c \circ A_{j}\left(e_{\ell}\right)=\left(1_{H} \otimes A_{j}\right) \circ \Delta\left(e_{\ell}\right)=\left(1_{H} \otimes A_{j}\right)\left(\sum_{n \in I} a_{\ell n} \otimes e_{n}\right)=\sum_{n \in I} a_{\ell n} \otimes A_{j}\left(e_{n}\right)=\sum_{n \in I} a_{\ell n} \otimes a_{n j}$.

(ii) Obviously, from $\left(\sigma \otimes 1_{E}\right) \circ \Delta=1_{E}$ we get, for $x \in E, x=\sum_{j \in I} \sigma\left(A_{j}(x)\right) e_{j}$. Hence $e_{\ell}=\sum_{j \in I} \sigma\left(A_{j}\left(e_{\ell}\right)\right) e_{j}=\sum_{j \in I} \sigma\left(a_{\ell_{j}}\right) e_{j}$ and $\sigma\left(a_{\ell j}\right)=\delta_{\ell j}$.

(iii) This follows readily from (i), (ii) and $m \circ\left(1_{H} \otimes \eta\right) \circ c=k \circ \sigma=m \circ\left(1_{H} \otimes \eta\right) \circ c$.

Lemma 5. Assume that $H$ admits a left integral $v$ such that $\langle v, e\rangle=1$. Put $\varphi=$ $v \circ m \circ\left(1_{H} \otimes \eta\right)$. For all $\ell, j \in I$, one has $\sum_{n \in I} \varphi\left(a_{\ell n} \otimes a_{n j}\right)=\delta_{\ell j}$.

Proof. Since $\varphi^{\circ} \mathrm{c}=\sigma$, from Lemma 4 one deduces that $\delta_{\ell_{j}}=\sigma\left(a_{\ell_{j}}\right)=\varphi^{\circ} c\left(a_{\ell_{j}}\right)=$ $\sum_{n \in I} \varphi\left(a_{\ell n} \otimes a_{n j}\right)$. 
Now assume that the duality $\left\langle H^{\prime}, H\right\rangle$ is separated and that $H$ admits a left integral $v$ such that $\langle v, e\rangle=1$. By Proposition 3 , we know that $\left(e_{e}^{\prime} \otimes e_{j}\right)^{\#} \in C(E)$, for all $\ell, j \in I$.

With (4) and by definition of $\left(e_{e}^{\prime} \otimes e_{j}\right)^{\#}$ one verifies that

$$
\left(e_{\ell}^{\prime} \otimes e_{j}\right)^{\#}\left(e_{i}\right)=\sum_{n \in I} \varphi\left(a_{i \ell} \otimes a_{j n}\right) e_{n}(i, j, \ell \in I) .
$$

REMARK 2. One deduces from (5) and Lemma 5 that there exists $\ell \in I$ such that $\left(e_{\ell}^{\prime} \otimes e_{\ell}\right)^{\#}$ is different from the null operator.

By a previous result, we know that the space $C(E)=E^{\prime} \hat{\otimes} E$ is isomorphic to $c_{0}\left(I, E^{\prime}\right)$. Let $u \in C(E)$; then $u=\sum_{\ell \in I} \psi_{\ell} \otimes e_{\ell}$ with $\psi_{\ell} \in E^{\prime}, \lim _{\ell} \psi_{\ell}=0$ and $\alpha_{0} \sup _{\ell \in I}\left\|\psi_{\ell}\right\| \leq$ $\|u\| \leq \alpha_{1} \sup _{\ell \in I}\left\|\psi_{\ell}\right\|$. Furthermore one has $u=\sum_{\ell \in I}{ }^{t} u\left(e_{\ell}^{\prime}\right) \otimes e_{\ell}$.

It is well known that one has the trace form $\operatorname{Tr}: E^{\prime} \otimes E \rightarrow K$ defined by $\operatorname{Tr}\left(x^{\prime} \otimes x\right)=\left\langle x^{\prime}, x\right\rangle$, which is linear and continuous with $|\operatorname{Tr}(u)| \leq\|u\|$. Here one obtains for $u=\sum_{\ell \in I} \psi_{\ell} \otimes e_{\ell} \in C(E)=E^{\prime} \hat{\otimes} E, \operatorname{Tr}(u)=\sum_{\ell \in I}\left\langle\psi_{\ell}, e_{\ell}\right\rangle=\sum_{\ell_{\in} I}\left\langle e_{\ell}^{\prime}, u\left(e_{\ell}\right)\right\rangle$.

Hence, for $u=\left(e_{\ell}^{\prime} \otimes e_{j}\right)^{\#} ; \ell, j \in I$; one has

$$
\operatorname{Tr}\left[\left(e_{\ell}^{\prime} \otimes e_{j}\right)^{\#}\right]=\sum_{n \in I} \varphi\left(a_{n \ell} \otimes a_{j n}\right) \quad(j, \ell \in I) .
$$

Definition. A complete Hopf algebra $H$ is called supple if $H$ is a pseudo-reflexive Banach space and if $\eta \circ \eta=1_{H}$.

EXAMPLEs. (1) $A P(G, K)$ and its complete sub-Hopf-algebras are supple.

(2) Any commutative (resp. cocommutative) complete Hopf algebra which is a pseudo-reflexive Banach space is supple.

LEMMA 6. Let $H$ be a supple complete Hopf algebra. If $H$ admits a left integral $v$ such that $\langle v, e\rangle=1$, then the map $\varphi=v \circ m \circ\left(1_{H} \otimes \eta\right)$ satisfies $\varphi(a \otimes b)=\varphi(b \otimes a)$, for all $a$, $b \in H$.

Proof. Since $\eta$ is an anti-endomorphism of the algebra $H$ and since $\eta \circ \eta=1_{H}$ implies $v \circ \eta=v$, one has for $a, \quad b \in H, \quad \varphi(a \otimes b)=v \circ m \circ\left(1_{H} \otimes \eta\right)(a \otimes b)=v(a \eta(b))=$ $v \circ \eta(a \eta(b))=v(b \eta(a))=\varphi(b \otimes a)$.

The following proposition strengthens Remark 2.

Proposition 4. Let $H$ be a supple complete Hopf algebra that admits a left integral $v$ such that $\langle v, e\rangle=1$. Let $E$ be a left Banach $H$-comodule which is a free Banach space with base $\left(e_{j}\right)_{j \in I}$. Then for each $\ell \in I$, the comodule endomorphism $\left(e_{\ell}^{\prime} \otimes e_{\ell}\right)^{\#}$ of $E$ is a completely continuous operator such that $\operatorname{Tr}\left[\left(e_{\ell}^{\prime} \otimes e_{\ell}\right)^{\#}\right]=1$.

Proof. Indeed, one deduces from (6), Lemmas 5 and 6 that $\operatorname{Tr}\left[\left(e_{\ell}^{\prime} \otimes e_{\ell}\right)^{\sharp}\right]=$ $\sum_{n \in I} \varphi\left(a_{n \ell} \otimes a_{\ell n}\right)=\sum_{n \in I} \varphi\left(a_{\ell n} \otimes a_{n \ell}\right)=1$

REMARK 3. In the same way, if $\ell \neq j$, then $\operatorname{Tr}\left[\left(e_{\ell}^{\prime} \otimes e_{j}\right)^{\#}\right]=\sum_{n \in I} \varphi\left(a_{n \ell} \otimes a_{j n}\right)=$ $\sum_{n \in I} \varphi\left(a_{j n} \otimes a_{n \ell}\right)=0$.

Let $E_{0}^{\prime}=E\left[e_{j}^{\prime}, j \in I\right]$ be the closed subspace of $E^{\prime}$ spanned by $\left(e_{j}^{\prime}\right)_{j \in I}$. The space 
$E_{0}^{\prime} \hat{\otimes} E$ is a closed subspace of $E^{\prime} \hat{\otimes} E=C(E)$. Since $\left(e_{j}^{\prime}\right)_{j \in I}$ is a base of $E_{0}^{\prime}$, each $u \in E_{0}^{\prime} \hat{\otimes} E$ can be written $u=\sum_{\ell \in I} \sum_{j \in I} \lambda_{\ell j} e_{j}^{\prime} \otimes e_{\ell}$ with $\lambda_{\ell j} \in K$, $\lim _{\ell} \sup _{j \in I}\left|\lambda_{\ell_{j}}\right|=0$ and for any $\ell \in I, \lim _{j}\left|\lambda_{\ell j}\right|=0$. One has $\operatorname{Tr}(u)=\sum_{\ell \in I} \lambda_{\ell \ell}$.

COROLlary 1. For each $u \in E_{0}^{\prime} \hat{\otimes} E$, one has $\operatorname{Tr}\left(u^{\#}\right)=\operatorname{Tr}(u)$. In particular, if $\operatorname{dim} E$ is finite, for each $u \in \mathscr{L}(E)$, one has $\operatorname{Tr}\left(u^{\#}\right)=\operatorname{Tr}(u)$.

Proof. Obviously, $\operatorname{Tr}\left(u^{\sharp}\right)=\sum_{\ell \in I} \sum_{j \in I} \lambda_{\ell j} \operatorname{Tr}\left[\left(e_{j}^{\prime} \otimes e_{\ell}\right)^{\#}\right]=\sum_{\ell \in I} \sum_{j \in I} \lambda_{\ell_{j}} \delta_{\ell_{j}}=\operatorname{Tr}(u)$.

COROLlary 2. Let $x \in E, x \neq 0$; there exists $x^{\prime} \in E^{\prime}$ such that $\left\langle x^{\prime}, x\right\rangle=1$ and $\operatorname{Tr}\left[\left(x^{\prime} \otimes x\right)^{\#}\right]=1$.

Let us recall that if $u \in C(E)$ then the Fredholm determinant of $u$ is $\operatorname{det}\left(1_{E}-t u\right)=$ $1+\sum_{q \geq 1}(-1)^{q} \operatorname{Tr}\left(\Lambda^{q} u\right) t^{q}$ and $\operatorname{det}\left(1_{E}-t u\right)$ is a power series of infinite radius of convergence; (cf. [4] and [10]). Furthermore $\operatorname{det}\left(1_{E}-t u\right) \cdot 1_{E}=\left(1_{E}-t u\right) P_{1}(t, u)$, where $P_{1}(t, u)$ is the Fredholm resolvent of $u$. Hence for $\lambda \in K, 1_{E}-\lambda u$ is invertible in $\mathscr{L}(E)$ if and only if $\operatorname{det}\left(1_{E}-\lambda u\right) \neq 0$.

With the operators as above, for instance for $u_{\ell}=\left(e_{\ell}^{\prime} \otimes e_{\ell}\right)^{\#}, \ell \in l$, one has

$$
\operatorname{det}\left(1_{E}-t u_{\ell}\right)=1-t+\sum_{q \geq 2}(-1)^{q} \operatorname{Tr}\left(\Lambda^{q} u_{\ell}\right) t^{q} .
$$

\section{Reducibility of Banach comodules.}

\section{III.1.1. Simple Banach comodules.}

Definimion. A left Banach $H$-comodule $E$ is called simple or topologically irreducible if $E$ is not the null space and does not contain any closed subcomodule different from (0) and $E$.

It follows immediately from Theorem 1 that, when $H$ is a pseudo-reflexive Banach space, any simple left Banach $H$-comodule is a vector space of countable type.

THEOREM 2. Let $H$ be a supple complete Hopf algebra that admits a left integral $v$ such that $\langle v, e\rangle=1$. Then any left Banach $H$-comodule that is not the null space contains at least a finite dimensional subcomodule different from $(0)$.

Proof. For this proof, we apply Riesz's decomposition theorem.

(a) Let $E$ be a left Banach comodule over the supple complete Hopf algebra $H$ with $E \neq(0)$. By Theorem 1, if $x \in E, x \neq 0$, then $x \in M=H^{\prime} \cdot x$; hence $M$ is different from (0). Furthermore $M$ is a Banach subcomodule of $E$ and is a Banach space of countable type. Therefore $M$ is a free Banach space (cf. [7]).

(b) Assume that $H$ admits a left integral $v$ such that $\langle v, e\rangle=1$. Hence by Proposition 4 , there exists a completely continuous operator $u$ which is an endomorphism of the comodule $M$ and such that $\operatorname{Tr}(u)=1$. It follows that $\operatorname{det}\left(1_{M}-t u\right)=1-t+\sum_{q \geq 2}(-1)^{q}$ 
$\operatorname{Tr}\left(\Lambda^{q} u\right) t^{q}$ is a non constant power series with infinite radius of convergence. According to the $p$-adic Weierstrass' factorization theorem, one has $\operatorname{det}\left(1_{M}-t u\right)=\prod_{q \geq 1} P_{q}$, where $P_{q}$ is a polynomical such that $P_{q}(0)=1, d \stackrel{\circ}{ } P_{q} \geq 1$ (see for example [1]). That is $\operatorname{det}\left(1_{M}-t u\right.$ ) has its zeros in a subfield of the algebraic closure $\tilde{K}$ of $K$. Following [10] one has the following results.

$\left(b_{1}\right) \quad$ First, $\operatorname{det}\left(1_{M}-t u\right)$ has a zero $\lambda \in K^{*}$.

Let $h \geq 1$ be the multiplicity of $\lambda$; one has $M=N(\lambda) \oplus F(\lambda)$ (Riesz's decomposition), where $N(\lambda)=\operatorname{ker}\left(1_{M}-\lambda u\right)^{h}$ and $\operatorname{dim} N(\lambda)=h$. However $\left(1_{M}-\lambda u\right)^{h}$ is a comodule endomorphism of $M$; therefore $N(\lambda)$ is a subcomodule of $M$ of finite dimension $h \geq 1$ and $N(\lambda)$ is a non-null finite dimensional subcomodule of $E$.

$\left(\mathrm{b}_{2}\right)$ Second, $\operatorname{det}\left(1_{M}-t u\right)$ has no zero in $K^{*}$.

Let $\zeta \in \tilde{K}$ be a zero of $\operatorname{det}\left(1_{M}-t u\right)$. Let $R(t)=1-\sum_{j=1}^{\ell} \gamma_{j} t^{j}$ be the polynomial of minimal degree such that $R\left(\zeta^{-1}\right)=0$ and $R(0)=1$. Setting $R(u)=1_{M}-\sum_{j=1}^{\ell} \gamma_{j} u^{j}=1_{M}-v$, we see that $v=\sum_{j=1}^{e} \gamma_{j} u^{j}$ is a comodule endomorphism of $M$ as well as a completely continuous operator.

Let $\zeta^{(2)}, \ldots, \zeta^{(\ell)}$ be the conjugates of $\zeta$ in $\tilde{K}$. The field $L=K\left[\zeta, \zeta^{(2)}, \ldots, \zeta^{(\ell)}\right]$ is a finite extension of $K$. Put $M_{L}=L \hat{\otimes}_{K} M=L \otimes_{K} M$; hence $u_{L}=1_{L} \otimes u$ is a completely continuous operator on $M_{L}$. Moreover, $\zeta \in L$ is a zero of $\operatorname{det}\left(1_{M_{L}}-t u_{L}\right)=\operatorname{det}\left(1_{M}-t u\right)$; hence $1_{M_{L}}-\zeta u_{L}$ is not invertible in $\mathscr{L}_{L}\left(M_{L}\right)$.

Since $R(t)=(1-\zeta t) \prod_{j=2}^{\ell}\left(1-\zeta^{(j)} t\right)$ and $R(u)=1_{M}-v$, one has

$$
R(u)_{L}=1_{M_{L}}-\sum_{j=1}^{\ell} \gamma_{j} 1_{L} \otimes u^{j}=1_{M_{L}}-\sum_{j=1}^{\ell} \gamma_{j} u_{L}^{j}=1_{M_{L}}-v_{L}=\left(1_{M_{L}}-\zeta u_{L}\right) \prod_{j=2}^{\ell}\left(1_{M_{L}}-\zeta^{(j)} u_{L}\right) .
$$

It follows that the completely continuous operator $v_{L}$ is such that $1_{M_{L}}-v_{L}=R\left(u_{L}\right)$ is not invertible in $\mathscr{L}_{L}\left(M_{L}\right)$. Consequently $1_{M}-v$ is not invertible in $\mathscr{L}(M)$; i.e. 1 is a zero of $\operatorname{det}\left(1_{M}-t v\right)$ with multiplicity $h^{\prime} \geq 1$. Therefore we have the Riesz decomposition $M=N(R) \oplus F(R)$, where $N(R)=\operatorname{ker}\left(1_{M}-v\right)^{h^{\prime}}=\operatorname{ker}\left(R(u)^{h^{\prime}}\right)$ and $\operatorname{dim} N(R)=h^{\prime} \geq 1$.

But $R(u)^{h^{\prime}}$ is a comodule endomorphism of $M$; hence $N(R)=\operatorname{ker}\left(R(u)^{h^{\prime}}\right)$ is a subcomodule of $M$ of finite dimension $h^{\prime} \geq 1$. Therefore $N(R)$ is a non-null finite dimensional subcomodule of $E$.

Remark 4. The subspace $F(\lambda)$ (resp. $F(R)$ ) of $M$ is also a Banach subcomodule of $E$.

THEOREM 3. Let $H$ be a supple complete Hopf algebra that admits a left integral $v$ such that $\langle v, e\rangle=1$. Then any simple left Banach $H$-comodule $E$ is finite dimensional.

Proof. This is obvious from Theorem 2. Indeed, let $x \in E, x \neq 0$; one has $\overline{H^{\prime} \cdot x}=$ $M \neq(0)$; hence $M=E$ and by Theorem 1, $E$ is a free Banach space. With the notations in the proof of Theorem 2, one has in the first case $E=N(\lambda)=\left\{x \in E / u(x)=\lambda^{-1} x\right\}$, $\operatorname{dim} E=h$ and $u=\lambda^{-1} 1_{E}$. In the second case, one has $E=N(R)=\{x \in E / v(x)=x\}$, $\operatorname{dim} E=h^{\prime}$ and $R(u)=0$. 
COROLLARY. (Schur's Lemma.) Under the above hypothesis on $H$, if $E$ is a simple left Banach $H$-comodule, then $\operatorname{End}_{\text {com }}(E)$ is a (skew) field of finite dimension $\leq(\operatorname{dim} E)^{2}$. Moreover, if $K$ is algebraically closed, then $\operatorname{End}_{\text {com }}(E)=K \cdot 1_{E}$ and if $K$ is of characteristic $p \neq 0$, then $(p, \operatorname{dim} E)=1$.

Proof. It suffices to observe that there exist $u \in \operatorname{End}_{\text {com }}(E)$ such that $\operatorname{Tr}(u)=1$. If $K$ is algebraically closed, one has $u=\lambda 1_{E}$, hence $\operatorname{Tr}(u)=1=\lambda \operatorname{dim} E$

\section{III.1.2. Reducibility of Banach comodules.}

Proposition 5. Let $H$ be a supple complete Hopf algebra that admits a left integral $v$ such that $\langle v, e\rangle=1$. Then any left Banach $H$-comodule $E$ that is a Banach space of countable type is a topological direct sum of simple comodules.

Proof. Indeed, by Theorem 2, $E$ contains finite subcomodules different from the null space. Hence $E$ contains a simple subcomodule. Let $W=\sum_{\ell \in S} V_{\ell}$ be the sum of all simple subcomodules of $E$. As in semi-simple module theory, there exists a subset $T$ of $S$ such that $W=\bigoplus_{\ell \in T} V_{\ell}$. Put $E_{0}=\bar{W}=\bigoplus_{\ell \in T} V_{\ell}$, the closure of $W$ in $E$. It is clear that $E_{0}$ is a Banach subcomodule of $E$.

On the other hand, since $E$ is a Banach space of countable type and $E_{0}$ is a closed subspace of $E$, for $0<\alpha<1$, there exists a linear projection of $E$ onto $E_{0}$ such that $\|p\| \leq$ $\frac{1}{\alpha}$ (cf. [7]). Therefore by the Corollary of Lemma 2 or Theorem 3 of [3] one has the direct sum of Banach comodules $E=E_{0} \oplus F_{0}$. If $F_{0}$ is different from (0), $F_{0}$ must contain a simple subcomodule $V$. Clearly $V$ is not contained in $E_{0}$; that contradicts the definition of $E_{0}$. Consequently $F_{0}=(0)$ and $E=E_{0}=\hat{\bigoplus}_{\ell \in T} V_{\ell}$.

THEOREM 4. Let $H$ be a supple complete Hopf algebra that admits a left integral $v$ such that $\langle v, e\rangle=1$. Then any left Banach $H$-module $E$ is a topological direct sum of simple comodules.

Proof. As above, put $W=\sum_{j \in I} V_{j}$, the sum of all simple subcomodules of $E$. There exists $J \subset I$ such that $W=\bigoplus_{j \in J} V_{j}$ (any simple subcomodule of $E$ is isomorphic to one of the $\left.V_{j}, j \in J\right)$.

Let $x \in E, x \neq 0$; the Banach subcomodule $M_{x}=\overline{H^{\prime} \cdot x}$ of $E$ being a Banach space of countable type, one has, by Proposition $5, x \in M_{x}=\bigoplus_{\ell \in T} V_{\ell}$, where $V_{\ell}$ is a simple subcomodule of $M_{x}$ and obviously of $E$. Hence for $\varepsilon>0$, there exists a finite subset $F$ of $T, x_{\ell} \in V_{\ell}$ for $\ell \in F$, such that $\left\|x-\sum_{\ell \in F} x_{\ell}\right\|<\varepsilon$. Since $\sum_{\ell \in F} x_{\ell} \in \bigoplus_{\ell \in F} V_{\ell} \subset W$, one has $x \in \bar{W}$ and $E=\bar{W}=\hat{\bigoplus}_{j \in J} V_{j}$.

REMARK 5. Let $\Omega$ be the family of the isomorphic classes of simple left Banach $H$-comodules. Let $E(\omega)$ be the isotypical component of $E$ for $\omega \in \Omega$, i.e. the sum of all simple subcomodules of $E$ belonging to $\omega$. It may happen that $E(\omega)=(0)$. One has 
$E(\omega)=\oplus\left(V_{j}, V_{j} \in \omega\right), W=\bigoplus_{\omega \in \Omega} E(\omega)$ and if $H$ satisfies the hypothesis of Theorem 4, then $E=\hat{\bigoplus}_{\omega \in \Omega} E(\omega)$.

III.2. Application to $H=A P(G, K)$. Let us recall that the complete Hopf algebra $A P(G, K)$ (as well as any of its complete Hopf subalgebras) is a supple Hopf algebra. A left integral $v$ over $A P(G, K)$, if it exists, such that $\langle v, 1\rangle=1$ is called an invariant mean.

From [3] we know that the category of left Banach $A P(G, K)$-comodules is in a bijective correspondence with the category of almost periodic linear representations of $G$.

The following theorem is a direct application of Theorems 3 and 4. One has an equivalent theorem for any complete Hopf subalgebra of $A P(G, K)$ as $A P_{\mathscr{T}}(G, K)$, $\mathscr{P P}(G, K)$ and $\mathscr{P} \mathscr{P}_{\mathcal{F}}(G, K)$ : the algebra $\mathscr{P} \mathscr{P}(G, K)$ is the subalgebra of the elements $f$ in $A P(G, K)$ such that $\left\{\gamma_{s} f, s \in G\right\}$ is relatively compact in $\mathscr{B}(G, K)$; if $\mathscr{T}$ is a group topology on $G, A P_{\mathscr{T}}(G, K)\left[\right.$ resp. $\left.\mathscr{P} \mathscr{P}_{\mathscr{T}}(G, K)\right]$ is the subalgebra of the functions $f$ in $A P(G, K)[$ resp. $\mathscr{P} \mathscr{P}(G, K)]$ such that $f$ is $\mathscr{T}$-continuous.

THEOREM 5. Assume that $A P(G, K)$ admits an invariant mean.

(i) Any topologically irreducible almost periodic linear representation is finite dimensional.

(ii) Let $U: G \rightarrow \mathscr{L}(E)$ be an almost periodic linear representation of $G$. The Banach space $E$ is a topological direct sum of irreducible $U$-invariant subspaces of $E$.

Note. Let $\Omega$ be the family of the classes of topologically irreducible almost linear representations. With the above hypothesis, one has $E=\bigoplus_{\omega \in \Omega} E(\omega)$ (cf. Remark 5).

Corollary 1. (Peter-Weyl Theorem). Assume that $A P(G, K)$ admits an invariant mean. Then the space $R_{b}(G, K)$ of the bounded representative functions of $G$ in $K$ is a dense subspace of $A P(G, K)$.

Proof. The left regular representation $\gamma$ of $G$ in $A P(G, K)$ is almost periodic $\left[\gamma_{s} f(t)=f\left(s^{-1} t\right)\right]$. It is clear that any $\gamma$-invariant finite dimensional subspace of $A P(G, K)$ is contained in $R_{b}(G, K)$.

Let $f \in A P(G, K), f \neq 0$ and $M_{f}=\overline{A P(G, K)^{\prime} \cdot f}$. One has $M_{f}=\hat{\bigoplus}_{\ell \in T} V_{\ell}$, where $V_{\ell}$ is $\gamma$-invariant and topologically irreducible. Hence $\operatorname{dim} V_{\ell}$ is finite and $V_{\ell} \subset R_{b}(G, K)$ for all $\ell \in T$. Moreover, as in the proof of Theorem 4, there exist a finite subset $F$ of $T$ and $\ell \in F, f_{\ell} \in V_{\ell}$, such that $\left\|f-\sum_{\ell \in F} f_{l}\right\|<\varepsilon$. Since $\sum_{\ell \in F} f_{\ell} \in \bigoplus_{\ell \in F} V_{\ell} \subset R_{b}(G, K)$, we have shown that $R_{b}(G, K)$ is dense in $A P(G, K)$.

Let $\omega \in \Omega$ be the class of the topologically irreducible almost periodic linear representation $(V, \rho)$ of $G$. With the hypothesis of Theorem 5 , one has $\operatorname{dim} V=n$ finite. Let $R(\rho)$ be the subspace of $A P(G, K)$ spanned by the coefficient functions of $\rho$; i.e. the functions $s \rightarrow\left\langle x^{\prime}, \rho(s) \cdot x\right\rangle$, where $x^{\prime} \in V^{\prime}$ and $x \in V$. One has $\operatorname{dim} R(\rho) \leq n^{2}$ and it is readily seen that $R(\omega)=R(\rho)$ depends only on $\omega$. Let $\check{\omega}$ be the class of $\left(V^{\prime}, \check{\rho}\right)$, where 
$\check{\rho}(s)={ }^{t} \rho\left(s^{-1}\right)$; then $\left(V^{\prime}, \check{\rho}\right)$ is irreducible and $R(\check{\omega})=\eta(R(\omega))$. Fix a base $\left(e_{j}\right)_{1 \leq j \leq n}$ of $V$ and let $\left(e_{j}^{\prime}\right)_{1 \leq j \leq n} \subset V^{\prime}$ be its dual base. Let us consider for $1 \leq j \leq n$ the linear map $A_{j}: V \rightarrow A P(G, K)$ defined by $A_{j}(x)(s)=\left\langle e_{j}^{\prime}, \rho\left(s^{-1}\right) \cdot x\right\rangle$. One has $\gamma_{s} \circ A_{j}=A_{j} \circ \rho(s)$ (directly or see Lemma 3). Since $A_{j}\left(e_{j}\right)(e)=1$, one has $\operatorname{ker} A_{j} \neq V$ and since $(V, \rho)$ is irreducible, $\operatorname{ker} A_{j}=(0)$, i.e. $A_{j}$ is injective. Put $H_{j}=A_{j}(V)$; the linear representations $(V, \rho)$ and $\left(H_{j}, \gamma\right)$ are equivalent. Hence $\left(H_{j}, \gamma\right) \in \omega$, for $1 \leq j \leq n$. It is readily seen that $\eta(R(\omega))=\sum_{j=1}^{n} H_{j}$ and there exists $J \subset[1, n]$ such that $\eta(R(\omega))=\bigoplus_{j \in J} H_{j}$. Moreover $\eta(R(\omega))$ is the isotypical component of $A P(G, K)$ corresponding to $\omega$. Therefore, if $\left(\omega_{1}, \ldots, \omega_{m}\right)$ is a finite subset of $\Omega$, then $\sum_{r=1}^{m} \eta\left(R\left(\omega_{r}\right)\right)=\bigoplus_{r=1}^{m} \eta\left(R\left(\omega_{\tau}\right)\right)$. It follows that $\sum_{\tau=1}^{m} R\left(\omega_{\tau}\right)=\bigoplus_{\tau=1}^{m} R\left(\omega_{\tau}\right)$

Since any finite dimensional almost periodic linear representation is reducible, we have proved the following result.

Corollary 2. Assume that $A P(G, K)$ admits an invariant mean. Then

$$
R_{b}(G, K)=\bigoplus_{\omega \in \Omega} R(\omega) \text { and } A P(G, K)=\hat{\bigoplus}_{\omega \in \Omega} R(\omega)
$$

Note. $R(\omega)$ is a subcogebra of $A P(G, K)$ for $\omega \in \Omega$.

Corollary 3. Assume that the group $G$ is commutative and that $A P(G, K)$ admits an invariant mean. If the field $K$ is algebraically closed, then $\Omega$ can be identified with $\operatorname{Hom}_{b}\left(G, K^{*}\right)=\hat{G}$, the bounded character group of $G$ and $A P(G, K)=\hat{\bigoplus}_{\chi \in \hat{G}} K \cdot \chi$.

Proof. The proof runs as in the classical case. Indeed, with the hypothesis on $A P(G, K)$, if $(V, \rho)$ is irreducible and $K$ is algebraically closed then $\operatorname{End}_{\rho}(V)=K \cdot 1_{V}$. Since $G$ is commutative, for $s \in G$, one has $\rho(s) \in \operatorname{End}_{\rho}(V)$, hence $\rho(s)=\chi(s) \cdot 1_{V}$ and $\chi$ is a bounded character of $G$ (which implies $|\chi(s)|=1, s \in G$ ). It follows that $\Omega=\hat{G}$. Since $R(\chi)=K \cdot \chi$, we have $A P(G, K)=\hat{\bigoplus}_{\chi \in \hat{G}} K \cdot \chi$ (compare with [8], [9]).

More generally one can prove the following result.

Corollary 4. Let $C A P(G, K)$ be the closed subalgebra of the central functions $f \in A P(G, K)$; i.e. $f\left(s t s^{-1}\right)=f(t), s, t \in G$. Assume that $A P(G, K)$ admits an invariant mean. Set for $(V, \rho) \in \omega, \chi_{\omega}^{u}(s)=\operatorname{Tr}(\rho(s) \circ u)$, where $u \in \operatorname{End}_{\rho}(V)$. Hence $\left\{\chi_{\omega}^{u}, \omega \in \Omega\right.$, $u \in \operatorname{End}_{\rho}(V)$ for a fixed $\left.(V, \rho) \in \omega\right\}$ is a total subset of the Banach space $C A P(G, K)$. Moreover, if $K$ is algebraically closed, setting $\chi_{\omega}(s)=\operatorname{Tr}(\rho(s))$ for a fixed $(V, \rho) \in \omega$, one has $C A P(G, K)=\bigoplus_{\omega \in \Omega} K \cdot \chi_{\omega}$.

Notes. (i) Let us say that $(G, K)$ is a.p.i.m. if $A P(G, K)$ admits an invariant mean $v$. Schikhof has given in [8], [9] the characterization of the a.p.i.m. pairs $(G, K)$ when $G$ is commutative and with the extra condition $\|v\|=1$. It remains to characterize all the a.p.i.m. pairs $(G, K)$.

(ii) If there exists an invariant mean $v$ on $A P(G, K)$, and putting for $f, g \in$ $A P(G, K),(f * g)(s)=\left\langle v, f \cdot \gamma_{s}(\eta(g))\right\rangle$, then $A P(G, K)$ is equipped with a new structure 
of Banach algebra, non unitary if $G$ is infinite. Can one use this algebra structure in the aim to establish the above results? In the case of $\mathscr{P P P}(G, K)$ see [6].

Remark 6. For any complete Hopf algebra $H$, one can define the representative subalgebra $\mathscr{R}(H)$ of $H$ similar to $R_{b}(G, K)$. If $H$ is supple and admits a left integral $v$ such that $\langle v, e\rangle=1$, then one has a translation of Theorem 5 and its Corollaries 1 and 2.

\section{REFERENCES}

1. Y. Amice, Les nombres p-adiques (P.U.F.-1975).

2. B. Diarra. Algèbres de Hopf et fonctions presque périodiques ultramétriques, to appear in Rivista di Matematica pura ed applicata $n^{\circ} 16$.

3. B. Diarra. Ultrametric almost periodic linear representations in $p$-adic Functional Analysis (Editorial Universidad de Santiago, Chile, 1994), 45-55.

4. L. Gruson. Théorie de Fredholm p-adique, Bull. Soc. Math. France 94 (1966), 67-95.

5. L. Gruson and M. van der Put. Banach spaces, Bull. Soc. Math. France, Mémoire 39-40 (1974), 55-100.

6. G. Rangan and M. S. Saleemullah. Banach algebra of $p$-adic valued almost periodic functions. in p-adic Functionnal Analysis, edited by J. M. Bayod, N. De Grande-De Kimpe and J. Martinez-Maurica (Marcel Dekker, New-York-1991), 141-150. 1978).

7. A. C. M. van Rooij: Non-archimedean functional analysis (Marcel Dekker, New York,

8. W. H. Schikhof. An approach to p-adic almost periodicity by means of compactoids. Report 8809-Departement of Mathematics, Catholic University (Nijmegen, 1988).

9. W. H. Schikhof. p-Adic almost periodic functions. Indag. Math. $1 \mathrm{n}^{\circ} 1$ (1990), 127-133.

10. J.-P. Serre. Endomorphismes complètement continus des espaces de Banach $p$-adiques Publ. Math. $\mathrm{n}^{\circ} 12$ (1962) (I.H.E.S. Paris), 69-85.

Mathematiques PURES

Complexe SCIENTIFIQue des CÉzeauX

63177 AUBIERE CEDEX

FRANCE

Fax: (33) 73.40 .70 .64

e-mail diarra@ucfma. univ-bpclermont.fr 